true for the study of localization in one dimension, for which the $T^{-2}$ dependence of the resistance predicted by Thouless remains to be observed. Copper wires, with thicknesses of $100 \AA$, widths of $1 \mu \mathrm{m}$, and lengths of $1 \mathrm{~cm}$ would seem to be well suited for that purpose.

We thank the Belgian Interuniversitair Instituut voor Kernwetenschappen for financial support, and the Nuclear Research Center, Mol, for technical assistance. We also acknowledge discussions with Professor A. Gilabert, University of Nice. One of us (L.V.d.d.) is a Research Fellow of the Belgian Interuniversitair Instituut voor Kernwetenschappen.

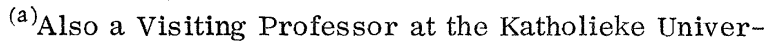
siteit, B-3030 Leuven, Belgium.

${ }^{1}$ D. J. Thouless, Phys. Rev. Lett. 39, 1167 (1977).

${ }^{2}$ E. Abrahams, P. W. Anderson, D. C. licciardello, and T. V. Ramakrishnan, Phys. Rev. Lett. 42, 673 (1979).

${ }^{3}$ P. W. Anderson, E. Abrahams, and T. V. Ramakrishnan, Phys. Rev. Lett. 43,718 (1979).
}

${ }^{4}$ G. J. Dolan and D. D. Osheroff, Phys. Rev. Lett. $\underline{43}$, 721 (1979).

${ }^{5}$ D. J. Bishop, D. C. Tsui, and R. C. Dynes, Phys. Rev. Lett. 44, 1153 (1980).

${ }^{6}$ N. Giordano, W. Gilson, and D. E. Prober, Phys. Rev. Lett. 43 , 725 (1979).

${ }^{7} \mathrm{P}$. Chaudhari and H. U. Habermeir, Phys. Rev. Lett. $44,40(1980)$.

${ }^{8}$ D. J. Thouless, Solid State Commun. 34, 683 (1980).

${ }^{9}$ J. L. Black, B. L. Giorffy, and J. Jäckles, Philos。 Mag. B 40, 331 (1979).

${ }^{10}$ B. L. Altshuler, A. G. Aronov, and P. A. Lee, Phys. Rev. Lett. 44, 1288 (1980).

${ }^{11}$ B. L. Altshuler, D. Khmel'nitzkii, A. J. Larkin, and P. A. Lee, Phys. Rev. B 22, 5142 (1980); T. F. Rosenbaum, K. Andres, G. A. Thomas, and P. A. Lee, to be published. We wish to thank Patrick Lee for making the result $F=(1 / x) \ln (1+x)$ available to us prior to publication.

${ }^{12}$ Deviation from $R_{\square} \propto 1 / d$ at small thickness indicates that films tend to become discontinuous with the apparition of macroscopic cracks and holes. The measured value of $R_{\square}$ is then enhanced above that of the material itself, which we expect should still vary as $1 / d^{2}$. For deviations from linearity less than $15 \%$, we have corrected the measured value of $R_{\square}$ as indicated by the arrows in Fig. 2 .

${ }^{13}$ L. Van den Dries, C. Van Haesendonck, Y. Bruynsraede, and $\mathrm{G}$. Deutscher, to be published.

\title{
Conductivity Cusp in a Disordered Metal
}

\author{
T. F. Rosenbaum, (a) K. Andres, G. A. Thomas, and P. A. Lee \\ Bell Laboratories, Murray Hill, New Jersey 07974
}

(Received 27 October 1980)

\begin{abstract}
A tendency toward a cusp at zero temperature in the electrical conductivity of Si crystals doped with $\mathrm{P}$ is observed. It is found that, within the metallic state, decreasing $\mathrm{P}$ concentration enhances the cusp and then rapidly changes its sign as a pseudogap opens. Such a cusp has been predicted for a disordered metal in which Coulomb interactions dominate the scattering.

PACS numbers: 71.45.-d, 71.50.+t, 72.15.Cz
\end{abstract}

The physics of disordered Fermi systems involves both the effects of localization and of Coulomb interactions, but their relative importance and interplay remain unresolved. Fundamentally different theories of localization ${ }^{1}$ and of electronic interaction $\mathrm{s}^{2,3}$ both agree with recent observations of a logarithmic dependence of the resistivity upon temperature and electric field in two-dimensional (2D) metals ${ }^{4}$ and with the behavior of thin wires. ${ }^{5}$ Hall-effect experiments ${ }^{6}$ on $2 \mathrm{D}$ electron inversion layers support the Coulomb-inter- action approach. In contrast, neither theory is supported by whisker and colloidal particle studies. ${ }^{7}$ Our results on dc conductivity and far-infrared transmission of 3D metallic samples of $\mathrm{Si}: \mathrm{P}$ appear to be explicable within the Coulombinteraction picture.

We have made four-probe resistance measurements of metallic, uncompensated samples of Si:P as a function of $T$ at frequencies $\sim 10 \mathrm{~Hz}$. Wires of $\mathrm{Au}: \mathrm{Sn}$ were spot welded to a freshly etched sample surface to make low-resistance, 
Ohmic contacts. The contacts were arranged linearly with an average length between voltage probes of $1 \mathrm{~mm}$ along an average cross-sectional area of $0.8 \times 0.5 \mathrm{~mm}^{2}$. Temperatures down to 50 $\mathrm{mK}$ were reached with use of a dilution refrigerator. Input power was confined to $<10^{-11} \mathrm{~W}$ and neither heating of the Si-crystal lattice nor thermal hysteresis was observed.

We have also made far-infrared transmission measurements using conventional Fourier-transform spectroscopy ${ }^{8}$ and crystals which were chemically thinned to about $1 \mu \mathrm{m}$. A sample area of $\sim 1 \mathrm{~mm}^{2}$ was illuminated by the far-infrared radiation.

The conductivity $\sigma$ as a function of $T$ is shown in Fig. 1. In the inset are the results for samples with the $\mathbf{P}$ density $n$ in units of $10^{18} \mathrm{~cm}^{-3}[\tilde{n}$ $\left.\equiv n /\left(10^{18} \mathrm{~cm}^{-3}\right)\right]$. The values of $n$ are determined from resistance measurements calibrated by neutron activation analysis. ${ }^{9}$ The $T$ dependence of $\sigma$ is small, but the low $-T$ region can be studied on a

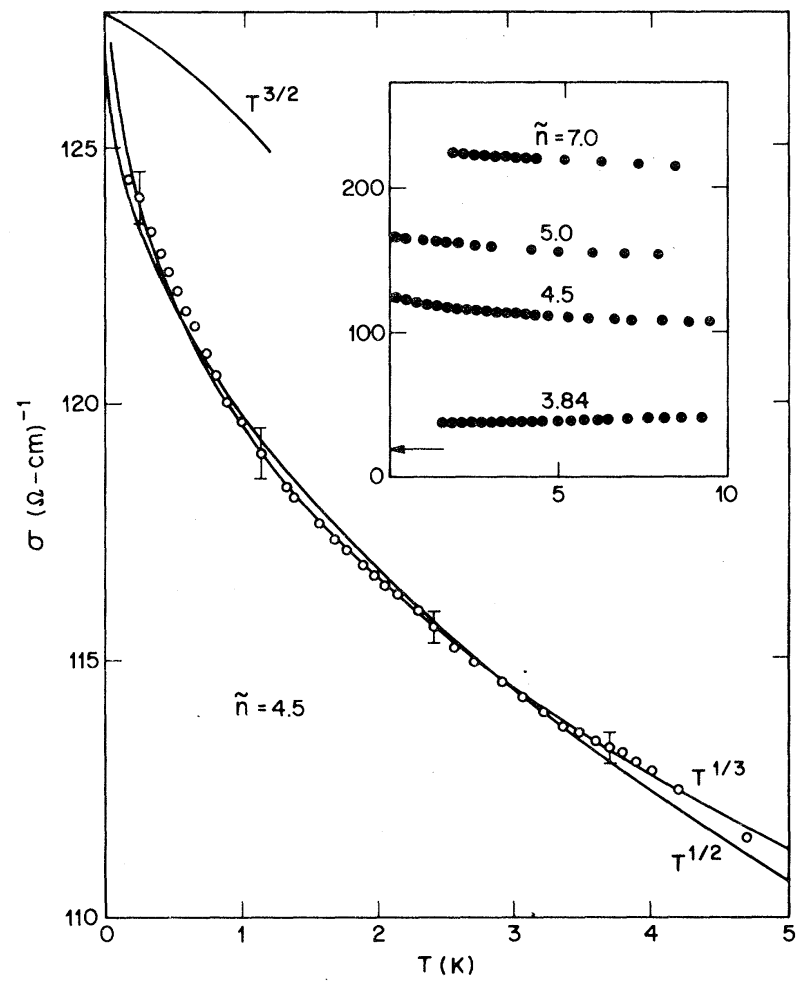

FIG. 1. Linear plot of conductivity $\sigma$ vs temperature $T$ for a representative metallic sample of Si:P, donor density $\tilde{n}=4.5\left(n\right.$ normalized to $\left.10^{18} \mathrm{~cm}^{-3}\right)$. The solid lines passing through the points are the functional forms $T^{1 / 3}$ and $T^{1 / 2}$. An alternative prediction, $T^{3 / 2}$, is also shown for comparison. In the inset are data for some samples without the expanded conductivity scale. The arrow indicates the minimum metallic conductivity. finer scale to display the conductivity cusp, as shown in the main part of Fig. 1. Studies by previous workers ${ }^{10}$ show similar small effects whose $T$ dependence has not been analyzed.

We have compared our results for $\sigma(T)$ with the simple power-law form

$$
\sigma(T)=\sigma(0)+m T^{\beta}
$$

A least-squares fit, varying $\sigma(0), m$, and $\beta$, gives values of $\beta$ in the range $0.3-0.7$. We have shown fits with both $\beta=\frac{1}{3}$ and $\frac{1}{2}$ as the solid lines in Fig. 1 to illustrate the uncertainty in $\beta$ for the sample with $\tilde{n}=4.5$. We confine the fits to $T<5 \mathrm{~K}$ in order to avoid contributions to $\sigma(T)$ from higher-order scattering processes. The cusplike shape is strikingly different from that of crystalline metals where the corresponding exponent $\beta$ is greater than 1 (producing no cusp) due, for example, to electron-electron scattering $(\beta=2)$ or to electron-phonon scattering $(\beta \geqslant 3)$. The negative coefficient $m$ shown in Fig. 1 has, however, the same sign as in crystalline metals.

For random metals we are aware of three estimates of the first-order corrections to $\sigma(0)$. First, if Coulomb interactions are neglected, the zero- $T$ scaling theory of localization ${ }^{1}$ can be extended ${ }^{11}$ to include phonon scattering, giving a correction of the form $m T^{3 / 2}$ with negative $m$. This shape, as shown in Fig. 1, is inconsistent with the data. Second, a renormalization-group analysis of the transition region, with the introduction of a temperature-dependent length scale, predicts $^{12}$ a correction $m T^{1 / 3}$ with positive $m$. This shape is consistent with measurements ${ }^{12}$ on $\mathrm{Ge}-\mathrm{Au}$ alloys and the exponent $\frac{1}{3}$ is consistent with our results. However, our observed negative sign of $m$ rules out this explanation for our samples with $k_{\mathrm{F}} l>1$, where $k_{\mathrm{F}}$ is the Fermi wave vector and $l$ is the mean free path.

A third approach is the formulation of Altshuler and Aronov, ${ }^{2}$ valid for $k_{\mathrm{F}} l \gg 1$, which predicts that electron-electron scattering in the presence of random impurities leads to a low-temperature conductivity

$$
\sigma(T)=\sigma(0)\left[1+f A\left(T / T_{\tau}\right)^{1 / 2}\right],
$$

where $A=0.72, T_{\tau}$ is a characteristic temperature given by

$$
T_{\tau} \equiv T_{\mathrm{F}}\left(k_{\mathrm{F}} l\right)^{3}=T_{\mathrm{F}}\left(\pi^{4} / 4\right)\left[\sigma(0) \hbar / e^{2}\right]^{3} / n,
$$

and $T_{\mathrm{F}}$ is the Fermi temperature. If the ThomasFermi screening length $\kappa^{-1}$ is much larger than the interparticle spacing $n^{-1 / 3}$, then only exchange terms need be included ${ }^{2}$ and $f=1$. How- 
ever, as $k_{\mathrm{F}} / \kappa$ gets smaller, Hartree terms also become important, giving ${ }^{3}$

$$
f=1-(3 / 2 x) \ln (1+x),
$$

where $x \equiv\left(2 k_{\mathrm{F}} / \kappa\right)^{2}$.

We have evaluated $x$ for $k_{\mathrm{F}} l>1$ using free-electron formulas ${ }^{13}$ which give $x=0.206 n^{1 / 3}$ for Si: P. For $n \simeq 10^{23} \mathrm{~cm}^{-3}, x$ is large and $f \rightarrow 1$. However, for $n$ in the region of our experiment, $x$ is small and $f$ becomes negative. Near the metal-insulator transition, the effectiveness of the screening degenerates rapidly, ${ }^{14}$ and $\kappa^{-1}$ tends to diverge as $n \rightarrow n_{c}$. Here $x$ again becomes large and $f$ changes sign a second time. In contrast, most dirty metals probably remain in the regime of large $x$ and positive $f$ since $n \sim 10^{23} \mathrm{~cm}^{-3}$.

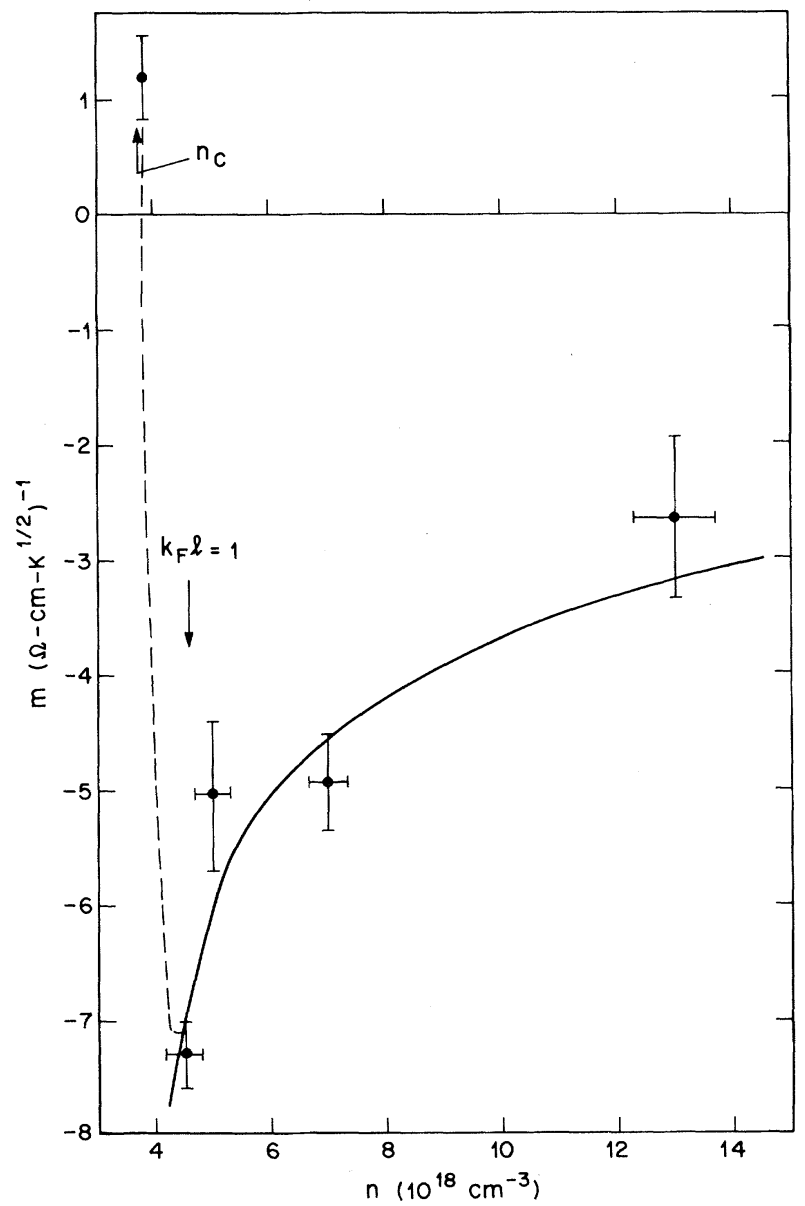

FIG. 2. Magnitude of the cusp when fitted by $T^{1 / 2}$ as a function of $n$. The solid line is a fit with the Coulombinteraction theory, Eqs. (2) -(4). The dashed line qualitatively shows the breakdown of screening for $k_{\mathrm{F}} l<1$, just above the metal-insulator transition which occurs at $n_{c}$.
To investigate the variation of $m$, defined by Eq. (1), we have carried out least-squares fits to $\sigma(T)$ with $\beta=\frac{1}{2}$. The results shown in Fig. 2 indicate that with decreasing $n$, the magnitude of the cusp grows, then drops rapidly and changes sign below $k_{\mathrm{F}} l=1$. The solid curve in Fig. 2 is the prediction of Eqs. (2)-(4) with use of $\sigma(0)$ as a function of $n$ determined by experiment ${ }^{15}$ and with use of the fitted value of $A=1.7 \pm 0.5$. The difference between this $A$ and the theoretical value may arise either because the theory is not accurate numerically for $k_{\mathrm{F}} l \approx 1$ or because Eq. (2) included contributions only from particle-hole scattering. Inclusion of particle-particle scattering (Ref. 3, Appendix B) produces an additional $\sqrt{T}$ term which will improve the agreement with experiment.

For $k_{\mathrm{F}} l<1$, the sign change of the cusp can be explained qualitatively by a divergence of $\kappa^{-1}$ as noted above and illustrated by the dashed line in Fig. 2, although Eqs. (2) -(4) are not valid. To investigate the regime where $k_{\mathrm{F}} l<1$ empirically,

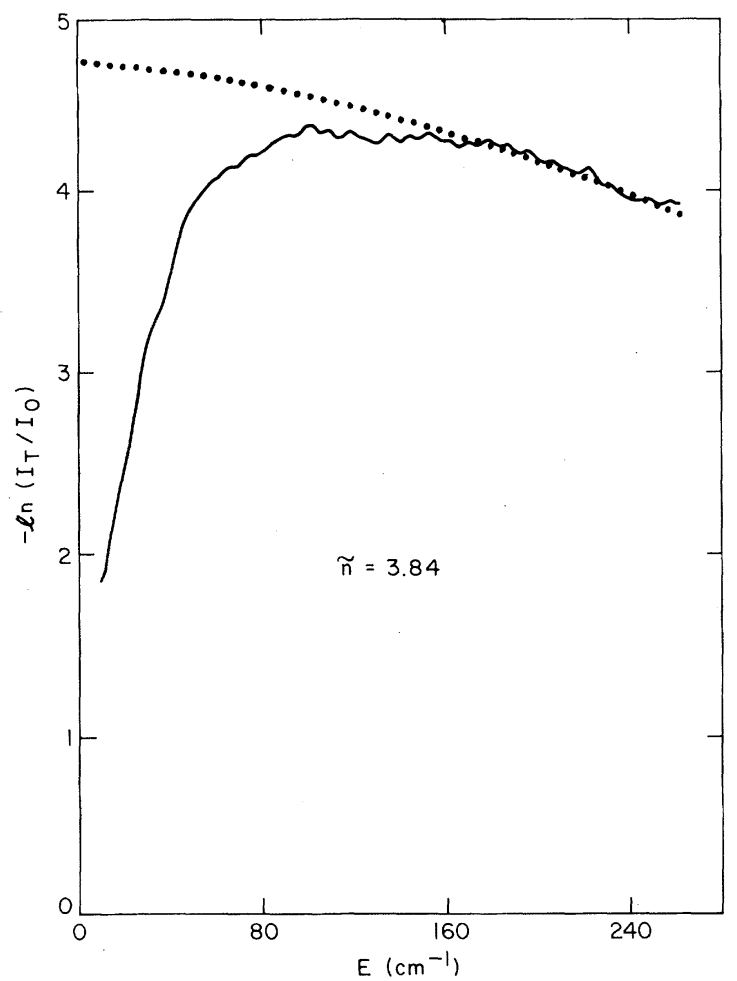

FIG. 3. Logarithmic plot of transmitted intensity vs far-infrared photon energy $E$ for a metallic sample with $\tilde{n}=3.84$. This logarithm varies approximately as $\sigma(\omega)$ and the factor-of-2 drop at low $E$ arises from a pseudogap in $\sigma(\omega)$. Drude behavior, fitted for $E \sim 200 \mathrm{~cm}^{-1}$, is shown as the dotted line for comparison. 
we have studied the far-infrared spectrum of another piece of the sample with $\tilde{n}=3.84$ and find a large drop at low frequencies as shown by the solid curve in Fig. 3. [In contrast, the theoretical Drude behavior for $\sigma(\omega)$ is shown as the dotted line.] The negative natural logarithm of the intensity transmitted through the Si:P sample $I_{T}$, normalized to that for a Si reference crystal $I_{0}$, is plotted. The behavior of $-\ln \left(I_{T} / I_{0}\right)$ as a function of frequency $\omega$ should be similar to that of $\sigma(\omega)$. The shape of $\sigma(\omega)$ is not necessarily the same as $\sigma(T)$, but $\sigma(T)$ increases roughly by a factor of 2 between 0 and $40 \mathrm{~K}$, as do the data between 0 and $40 \mathrm{~cm}^{-1}$. We measure $\hbar \omega \gg k T$ and find that here, with $T \sim 2 \mathrm{~K}$, the data are independent of $T$ to the level of our accuracy. The rapid development of this pseudogap, even though the sample remains a metal [as indicated by $\sigma(T)$ ], provides further evidence for the breakdown of metallic screening for $k_{\mathrm{F}} l<1$.

To our knowledge Fig. 3 is the first direct observation of a metallic pseudogap in $\sigma(\omega)$. This effect, involving particle-hole excitation, differs from a pseudogap in the single-particle density of states which could be measured by tunneling. ${ }^{16}$ The metallic pseudogap that we have observed can be described qualitatively, as noted above, by extrapolating ${ }^{12}$ the interaction theory for dis ordered systems ${ }^{3}$ into the region of the transition. Alternatively, a strong dip in the density of states is also predicted in the same region for a half-filled Hubbard band. ${ }^{17}$ The resulting metal is expected to be strongly correlated ${ }^{18}$ and evidence of this correlation has been found in the magnetic susceptibility. ${ }^{19}$ The metallic pseudogap is expected within this picutre only for uncompensated samples (with half-filled bands) such as ours, and not for compensated samples.

In conclusion, we have observed behavior that can be explained if Coulomb interactions dominate $\sigma(T)$ and $\sigma(\omega)$ in a disordered metal.

We would like to acknowledge helpful discussions with P. W. Anderson, R. N. Bhatt, E. I. Blount, and T. M. Rice, and technical assistance from Frank DeRosa.

\footnotetext{
(a)Also at Joseph Henry Laboratory, Princeton University, Princeton, N.J. 08544.

${ }^{1}$ E. Abrahams, P. W. Anderson, D. C. Licciardello, and T. V. Ramakrishnan, Phys. Rev. Lett. $\underline{42}, 673$
}

(1979), and references therein; Y. Imry, Phys. Rev. Lett. 44,469 (1980).

${ }^{2}$ B. L. Altshuler and A. G. Aronov, Zh. Eksp. Teor. Fiz. 77, 2028 (1979) [Sov. Phys. JETP 50, 968 (1979)], and Pis'ma Zh. Eksp. Teor. Fiz. 27, 700 (1978) LJETP Lett. 27, 662 (1978)], and Solid State Commun. 36, 115 (1979).

${ }^{3}$ B. L. Altshuler, A. G. Aronov, and P. A. Lee, Phys. Rev. Lett. 44, 1288 (1980); B. L. Altshuler, D. Khmelnitzkii, A. I. Larkin, and P. A. Lee, Phys. Rev. B 22, $5142(1980)$.

${ }^{4}$ G. J. Dolan and D. D. Osheroff, Phys. Rev. Lett. 43, 721 (1979); D. J. Bishop, D. C. Tsui, and R. C. Dynes, Phys. Rev. Lett. 44,1153 (1980).

${ }^{5}$ N. Giordano, W. Gilson, and D. E. Prober, Phys. Rev. Lett. $\underline{43}, 725$ (1979); P. Chaudhari and H.-V. Habermeier, Phys. Rev. Lett. 44, 40 (1980); P. Chaudhari, A. N. Broers, C. C. Chi, R. Laibowitz, E. Spiller, and J. Viggiano, Phys. Rev. Lett. 45, 930 (1980).

${ }^{6}$ D. J. Bishop, D. C. Tsui, and R. C. Dynes, Phys. Rev. Lett. 46, 360 (1981); M. J. Uren, R. A. Davies, and M. Pepper, to be published.

${ }^{7}$ D. R. Overcash, R. A. Ratnam, M. J. Skove, and E. P. Stillwell, Phys. Rev. Lett. 44, 1348 (1980); J. C. Garland, W. J. Gully, and D. B. Tanner, Phys. Rev. B 22, 507 (1980).

${ }^{8}$ G. A. Thomas, M. Capizzi, and F. DeRosa, Philos. Mag. B42, 913 (1980); G. A. Thomas, M. Capizzi, F. DeRosa, R. N. Bhatt, and T. M. Rice, Phys. Rev. $\mathrm{B}$ (to be published).

${ }^{9}$ F. Mousty, P. Ostoja, and L. Passari, J. Appl. Phys. $\underline{45}, 4576$ (1974); T. F. Rosenbaum, K. Andres, and G. A. Thomas, Solid State Commun. 33, 663 (1980).

${ }^{10} \mathrm{C}$. Yamanouchi, K. Mizuguchi, and W. Sasaki, J. Phys. Soc. Jpn. 22, 859 (1967); W. Sasaki and R. De Bruyn Ouboter, Physica (Utrecht) 27, 877 (1961); Y. Katayama and S. Tanaka, Phys. Rev. 153, 873 (1967). ${ }^{11} \mathrm{P} . \mathrm{W}$. Anderson, private communication.

${ }^{12}$ W. L. McMillan, private communication; B. W. Dodson, W. L. McMillan, J. M. Mochel, and R. C. Dynes, Phys. Rev. Lett. 46, 46 (1981).

${ }^{13}$ See, for example, C. Kittel, Introduction to Solid State Physics (Wiley, New York, 1953).

${ }^{14}$ T. M. Rice and W. F. Brinkman, in Critical Phenomena in Alloys, Magnets, and Superconductors, edited by R. E. Mills, E. Asher, and R. I. Jaffe (McGrawHill, New York, 1971), p. 593.

${ }^{15}$ T. F. Rosenbaum, K. Andres, G. A. Thomas, and R. N. Bhatt, Phys. Rev. Lett. 45, 1723 (1980).

${ }^{16}$ E. L. Wolf, R. H. Wallis, and C. J. Adkins, Phys. Rev. B 12, 1603 (1975), and references therein. Tunneling results on granular Al films provide support for Coulomb interactions: R. C. Dynes and J. P. Garno, Phys. Rev. Lett. 46, 137 (1981).

${ }^{17}$ N. F. Mott, The Metal-Insulator Transition (Taylor and Francis, London, 1974), p. 44.

${ }^{18}$ W. F. Brinkman and T. M. Rice, Phys. Rev. B 2 , 4302 (1970).

${ }^{19}$ J. D. Quirt and J. R. Marko, Phys. Rev. Lett. 26 , 318 (1973); S. Geschwind, R. Romestain, and G. E. Devlin, J. Phys. (Paris), Colloq. 37, C4-313 (1976). 